\title{
Automated Mango Fruit Grading System Using Fuzzy Logic
}

\author{
Yeong Kin Teoh ${ }^{1}$, Suzanawati Abu Hasan ${ }^{1}$ \& Suraiya Sauddin@Sa'duddin ${ }^{1}$ \\ ${ }^{1}$ Mathematics and Statistics Department, Universiti Teknologi MARA (Perlis), Arau, Perlis, Malaysia \\ Correspondence: Yeong Kin Teoh, Mathematics and Statistics Department, Universiti Teknologi MARA (Perlis), \\ 02600 Arau, Perlis, Malaysia. Tel: 60-19-359-8485. E-mail: ykteoh@perlis.uitm.edu.my
}

Received: October 8, 2013 Accepted: November 13, 2013 Online Published: December 15, 2013

doi:10.5539/jas.v6n1p41

URL: http://dx.doi.org/10.5539/jas.v6n1p41

\begin{abstract}
This paper concentrates on the size of mango fruit. Mangoes grading by humans in current agricultural industry are subjective, inconsistent and inefficient because there is an individual difference in visual inspecting which is affected by environment, physical and psychological conditions. In this paper, fuzzy logic is used to create a novel grading method. A membership function and fuzzy rules are generated from training instances based on minimum entropy formulas. Computer and Red Green and Blue (RGB) fiber optic sensor are used to examine and clarify data corresponding to human judgment and intelligence. A total of $77.78 \%$ of accuracy is achevied under the proposed method which capable of differentiating three different grades of mango. This paper offers a competent practice and capable to be applied to improve and standardize the current mango fruit grading system.
\end{abstract}

Keywords: classification, fuzzy logic, mango grading, RGB fiber optic sensor

\section{Introduction}

Mango fruit (Mangifera Indica) is native to South Asia. Thailand is the main producer of the mangoes in South East Asia with production of 2.55 million tonnes in 2010-11 (Statistical Division, FAO, 2013). Other than Thailand, there are also other countries that are supplying mangoes to the world such as Indonesia, Australia and Philippines. According to Department of Statistics Malaysia, production of mango in Malaysia in 2011 is 21,021.5 tonnes with an estimation value of 111 million ringgit (Fruit Crops Statistic, Department of Agriculture Peninsular Malaysia, 2011). Mango is a tropical fruit that has high demand every year and also gets a good price in the world market. It is important to select the fruit with the right degree of maturation in order to ensure the supply of high-quality fruits.

Over the past 20 years, fruit categorizations in agriculture industry have rapidly changed from traditional human grading system to automated grading system. In order to improve agriculture sector, implementation of Information and Communication Technology (ICT) was proposed and the idea of using ICT in agriculture sector initiates the development of an automated grading system (Mustafa et al., 2009). Several features such as size, appearance and flavor are the key indicators to determine the grade or quality of the agriculture products. In agriculture industry, many companies are using automated grading technique in fruits such as peaches and oranges (Blasco et al., 2007). Other fruit such as apple also using automated grading technique (Kavdir \& Guyer, 2003). Different fruits have their different attributes to be considered when they go through the grading process. Size, color, stem location and detection of external blemishes are the chosen characteristics for oranges, peaches and apples (Blasco et al., 2003). Size, color and shape have been considered in the grading process of tomato (Jahns et al., 2001) and strawberry (Liming \& Yanchao, 2010).

In Malaysia, the present mango grading process is by using the human expert to grade mangoes. Human expert means that the grading process is done by eyes and hands of the experts. This method creates some disadvantages such as lack of objectivity, accuracy and efficiency. In this study, we applied fuzzy technique in order to determine the size of the mangoes based on RGB digital fiberoptic sensor and hence graded them accordingly.

\section{Materials and Methods}

This proposed grading system relies on the sizes of the mangoes. Thus, size measurement plays a vital position in creating this grading system. There are seven processing level involved in this study. The flow of each processing level is summarized in Figure 1. 


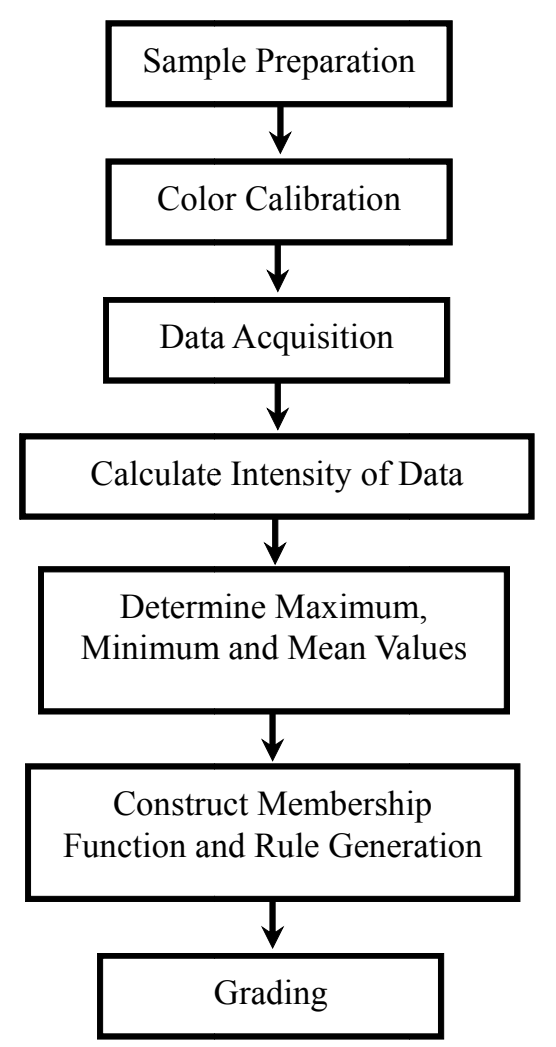

Figure 1. The flowchart of processing level

\section{A. Sample Preparation}

The type of mangoes being used in this study is Chokanan. They are collected from Tobiar Gold Mango Farm and graded by experts immediately after the mangoes collected. 150 mangoes are used in order to carry out this study.

\section{B. Color Calibration}

In order to fulfill the objective in obtaining the sizes of the mangoes by using the RGB fiberoptic sensor, red color calibration is used. One scale of color (red) acts as a color reference that can give the RGB sensor value that will be used in the next step.

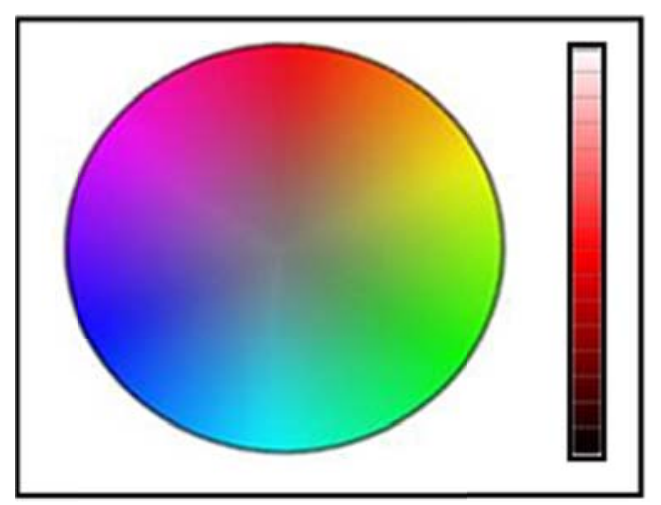

Figure 2. HSL reference chart and color calibration 


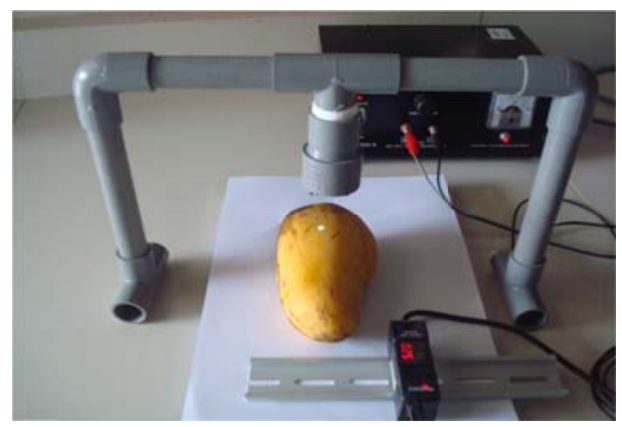

Figure 3. RGB fiberoptic sensor

\section{Data Acquisition}

Length, width and height for each of the mangoes are measured. An adjustable box is used in order to obtain the measurement. In that particular adjustable box, there is a ruler to measure the mango fruit. Once the measurements are obtained, the sensor that uses only red color will be pointed out at the color scale based on the measurements obtained in centimeters. This process also known as calibration process.

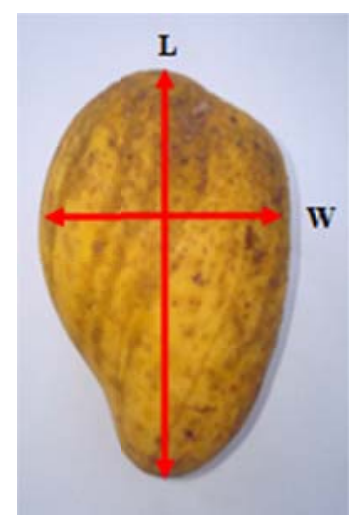

Figure 4. Dimension of Mango

\section{Calculation of Intensity of Data}

The intensity of data is the value obtained from the RGB sensor. The red sensor of RGB is pointed out at the adjustable box. Once the red sensor is being pointed out at the adjustable box, there is a given value that indicates the intensity of the data.

\section{E. Determination of Maximum, Minimum and Mean Values}

Maximum, minimum and mean values are identified for each size of the mango in order to create a membership functions for each category of mango. Using a minimum entropy formulation, membership functions are created for each category which is small, medium and large. Membership functions must be created because it showed the overlapping of the size of the mango from the different categories.

\section{F. Construct Membership Function and Rule Generation}

Minimum entropy formulation is used to obtain the membership function in grading the mangoes. From the membership function obtained, rules of inference were created.

\section{G. Grading}

Size is the most common feature of the post-harvest fruit handling nowadays. In this study, mangoes are graded in terms of size. If the size is large, then the mango is graded as Grade A. Next, if the size is medium, then the mango is in Grade B. Finally, if the mango is small, then the mango is in Grade C. Fuzzy logic is implemented in order to classify the mangoes into Grade A, Grade B and Grade C categories. Fuzzy logic is chosen because it offers a fine approach when come to handle subjective criteria which often happen in grading process. In current scenario, 
mango grading is completed by using human expert which is totally based on the experience. In order to standardize the grading process, an improvement model needs to be introduced.

There are three inputs (Length, Width and Height) and one output (Size) in the grading system as shown in Figure 5. 27 rules are formed to categorize the mango grade.

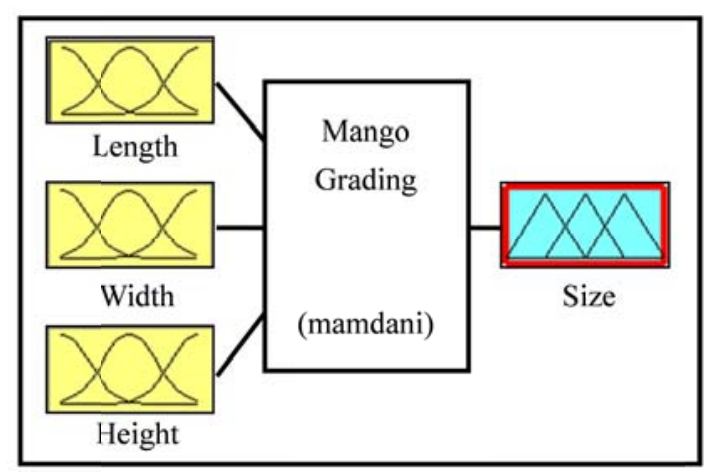

Figure 5. The fuzzy inference system

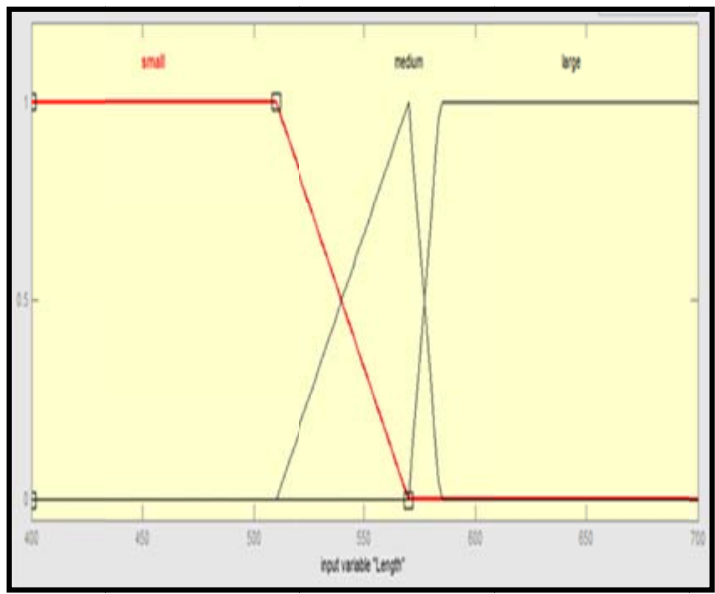

Figure 6. Membership function of length input

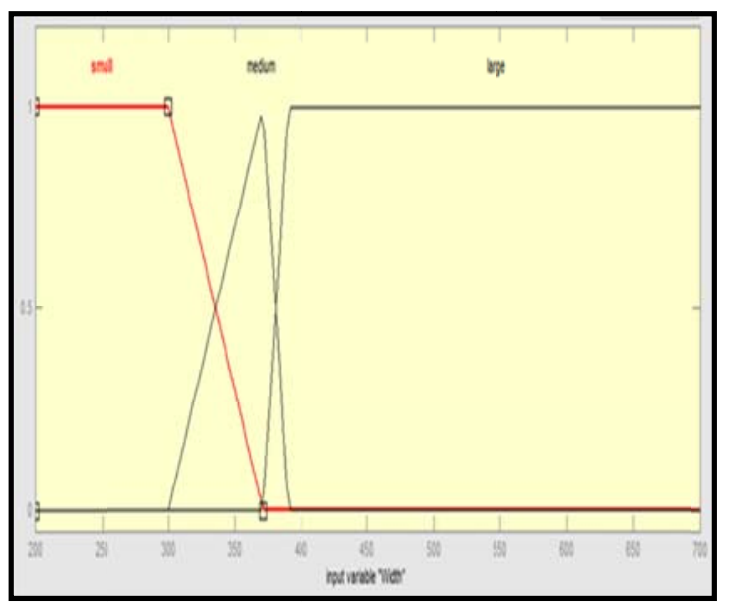

Figure 7. Membership function of width input 


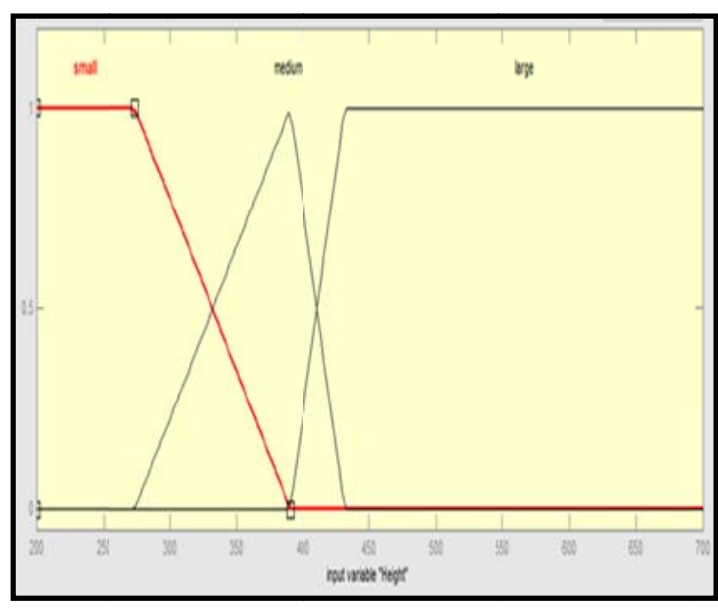

Figure 8. Membership function of height input.

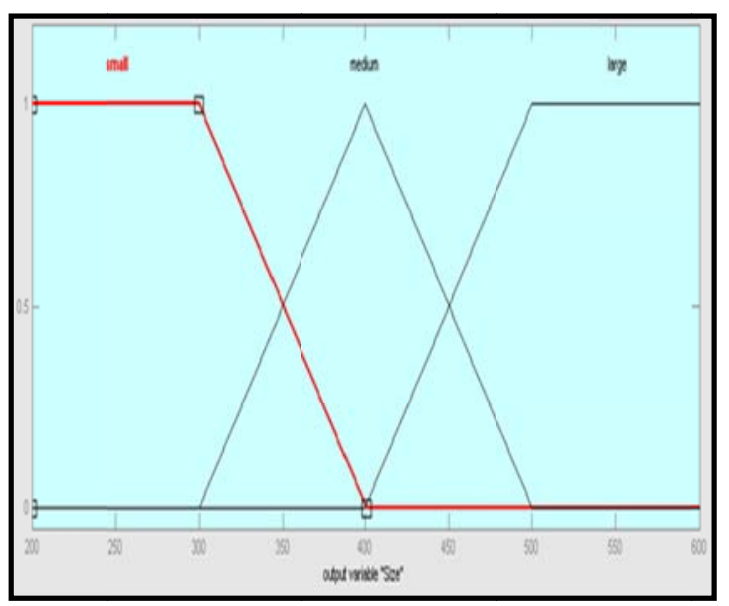

Figure 9. Membership function of size output

Figure 9 is the membership function resulted from the combination of membership functions of length, width and height as shown in Figure 6, Figure 7 and Figure 8 respectively. All of these membership functions were used to develop rules of inference. All together there are 27 rules statements have been produced by the fuzzy sets to classify the mango's category. Examples of the rules are illustrated as follows:

Table 1. Fuzzy Sets Rules for Classification

\begin{tabular}{cccc}
\hline Rule 3 & Rule 8 & Rule 13 & Rule 18 \\
\hline If Length is small & If Length is small & If Length is medium & If Length is medium \\
And Width is small & And Width is large & And Width is medium & And Width is large \\
And Height is large & And Height is medium & And Height is small & And Height is large \\
Then Size is small & Then Size is medium & Then Size is medium & Then Size is large \\
\hline
\end{tabular}

\section{Results and Discussion}

The developed mango grading system is able to categorize the mango into different grades. In order to establish an appropriate range of RGB value for each grade, the mean value of each grade is calculated and compared with the RGB values. 
Table 2. Measurement of mango size

\begin{tabular}{cc}
\hline Size & Range \\
\hline Small & $x<300$ \\
Medium & $300 \leq x \leq 500$ \\
Large & $x>500$ \\
\hline
\end{tabular}

Table 2 shows the output of the grading sizes of mango. In this study, classification of the mango size is based on the table above. The range stated in the Table 2 was the intensity value shown in the membership function in Figure 9.

A case of misclassification of mango by human grader is given in Figure 10. The mango is classified as medium (Grade B) by human grader but the grading program classified it as large grade (Grade A). This dilemma happens because the sizes (length, width and height) of the mango lie between medium and large categories. The value of length lies in the range of large category whereas the values of width and height lie in the range of medium category and lead to the misclassification.

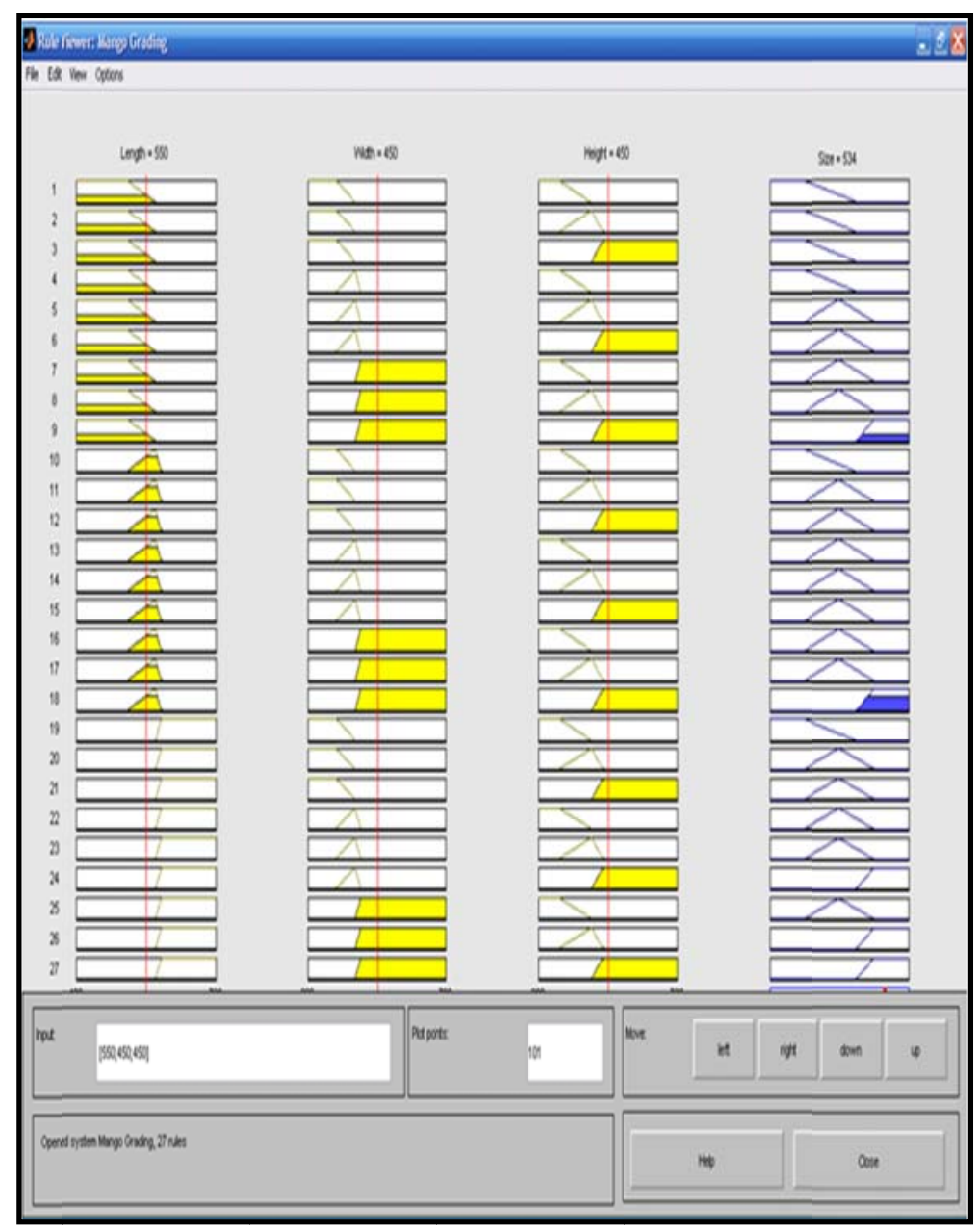

Figure 10. The misclassification result from rule viewer

An improved range for each measurement (length, width and height) needs to be achieved with the aim of improving the grading system performance. An additional feature such as color that fit in between the categories need to be added in order to obtained better system performance.

The results obtained using RGB sensor and membership functions are compared to the human graders in term of 
accuracy. It is clear that fuzzy logic grading system has overcome traditional human grading system in term of accuracy and efficiency in grading the mango because the proposed system accomplished a $77.78 \%$ of accuracy in all three categories.

\section{Conclusion}

This research has established that by implementing fuzzy logic together with several computer software and minimum entropy formulas, the accuracy of mango grading is high. This technique begins with taking the data of mango and inserting them into the program and fuzzy logic technique was implemented in grading process. The mango is categorized based on the RGB intensity values.

The main objective of this project in designing fuzzy logic is the determination of membership function in order to classify and to grade mango fruits. From the membership function, the range of size for mango fruits was obtained. In this study, an inductive reasoning method in designing fuzzy membership functions was used. This approach is based on minimizing entropy function which is partitioning a threshold line between three variables of data: length, width and height of mango fruit. The results show membership functions obtained are shaped from the threshold. On the other hand, this project also applied the IF-THEN Rules and Mamdani Implication to investigate the relationships which exist between the variables.

\section{Acknowledgements}

I express my profound gratitude to Azhar for providing the mango sample and his full assistance throughout the research project. The mangoes are taken from the Tobiar Gold mango farm located at Tobiar, daerah Pendang, Kedah, Malaysia. Earnest appreciation also goes to Razak bin Mansor and Associate Professor Dr. Mahmod bin Othman for their assistance in guiding us the way to apply fuzzy technique in my research.

\section{References}

Blasco, J., Aleixos, N., \& Molto, E. (2003). Machine Vision System for Automatic Quality Grading of Fruit. Biosystems Engineering, 85(4), 415-423. http://dx.doi.org/10.1016/S1537-5110(03)00088-6

Blasco, J., Aleixos, N., Gomez, J., \& Molto, E. (2007). Citrus sorting by identification of the most common defects using multispectral computer vision. Journal of Food Engineering, 83(3), 384-393. http://dx.doi.org/10.1016/j.jfoodeng.2007.03.027

Fruit Crops Statistic, Department of Agriculture Peninsular Malaysia. (2011). Retrieved from http://www.doa.gov.my/c/document_library/get_file?uuid=37c24b5a-01fc-44f0-961e-d2c987cf12a5\&group $\mathrm{Id}=38371$

Jahns, G., Nielsen, H. M., \& Paul, W. (2001). Measuring Image Analysis Attributes and Modelling Fuzzy Consumer Aspects for Tomato Quality Grading. Computers and Electronics in Agriculture, 31, 17-29. http://dx.doi.org/10.1016/S0168-1699(00)00171-X

Kavdir, I., \& Guyer, D. E. (2003). Apple Grading Using Fuzzy Logic. Turkish Journal of Agriculture and Forestry, $27,375-382$.

Liming, X., \& Yanchao, Z. (2010). Automated Strawberry Grading System Based on Image Processing. $\begin{array}{lllll}\text { Computers and Electronics in } & \text { Agriculture, } & 71(\mathrm{~S} 1), & \text { S32-S39. }\end{array}$ http://dx.doi.org/10.1016/j.compag.2009.09.013

Mustafa, N., Ahmed, S., Ali, Z., Yit, W., Abidin, A., \& Sharrif, Z.. (2009). Agricultural procedure sorting and grading using support vector machines and fuzzy logic. IEEE International Conference on Signal and Image Processing Applications, 18, 391-396.

Statistical Division, Food and Agricultural Organization of United Nations (FAO). (2013). Retrieved from http://www.fao.org/statistics/en

\section{Copyrights}

Copyright for this article is retained by the author(s), with first publication rights granted to the journal.

This is an open-access article distributed under the terms and conditions of the Creative Commons Attribution license (http://creativecommons.org/licenses/by/3.0/). 